\title{
Calcinosis and Rapidly Destructive Arthropathy Associated with Anti-Jo1 Antibody
}

LIT HIANG LEE, MRCP (UK), Specialty Registrar; ASHA SRIKANTH, MRCP (UK), Consultant; ANINDYA GUPTA, FRCP (UK), PhD, Consultant, Department of Rheumatology, Queen's Medical Centre, Nottingham, UK. Address correspondence to Dr. L.H. Lee, Department of Rheumatology, Queen's Medical Centre, Derby Road, Nottingham, NG7 2UH, UK. E-mail: L.H.Lee@doctors.org.uk. J Rheumatol 2015;42:547-8; doi:10.3899/jrheum.140738

We report here a rare case of marked calcinosis and rapidly destructive arthropathy associated with anti-Jo1 antibody.

A 74-year-old white woman presented to the rheumatology clinic in 2008 with a 5-year history of polyarthralgia affecting her left hip, right elbow, left shoulder, and hands. Hand and elbow radiographs (Figure 1) showed destructive arthropathy thought to be attributable to severe erosive osteoarthritis or crystal arthritis.

Repeat radiographs in 2010 showed extensive periarticular calcinosis and complete destruction of the right elbow joint.

Similar changes were noted on the repeat hand radiograph in 2012 (Figure 2). Immunological profile revealed strongly positive antinuclear antibody in a nucleolar pattern and anti-Jo1 antibody. Muscle biopsy showed numerous atrophic fibers, but no active inflam-

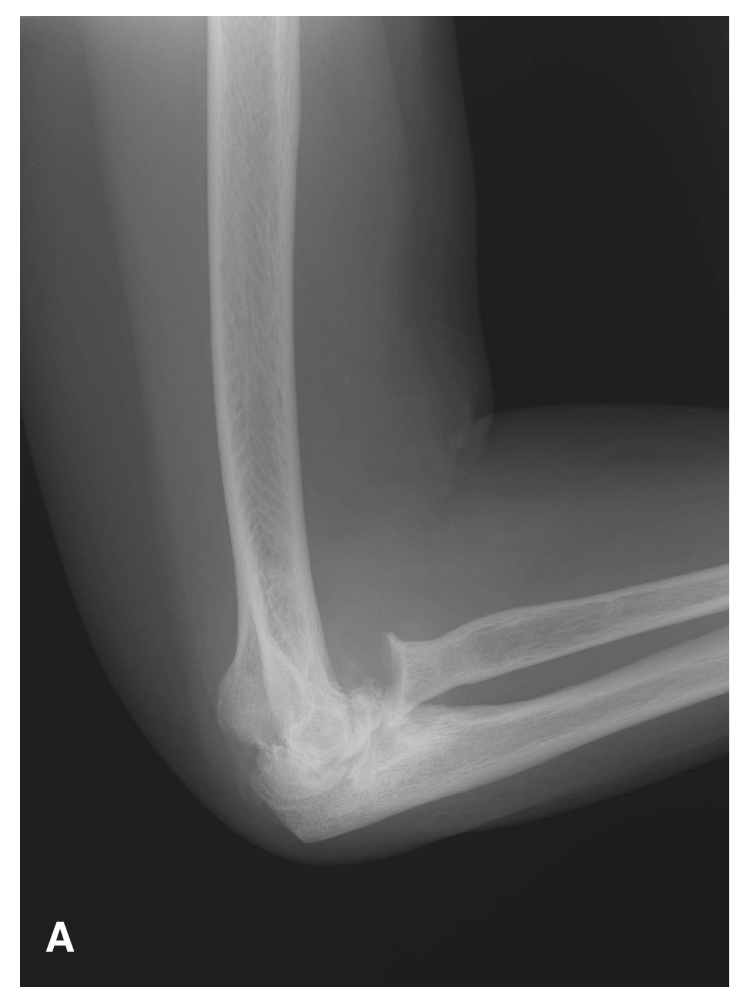

Figure 1. Radiographs of the right elbow joint taken in (A) 2008 and (B) 2010 demonstrate pronounced progression of joint destruction and calcinosis.

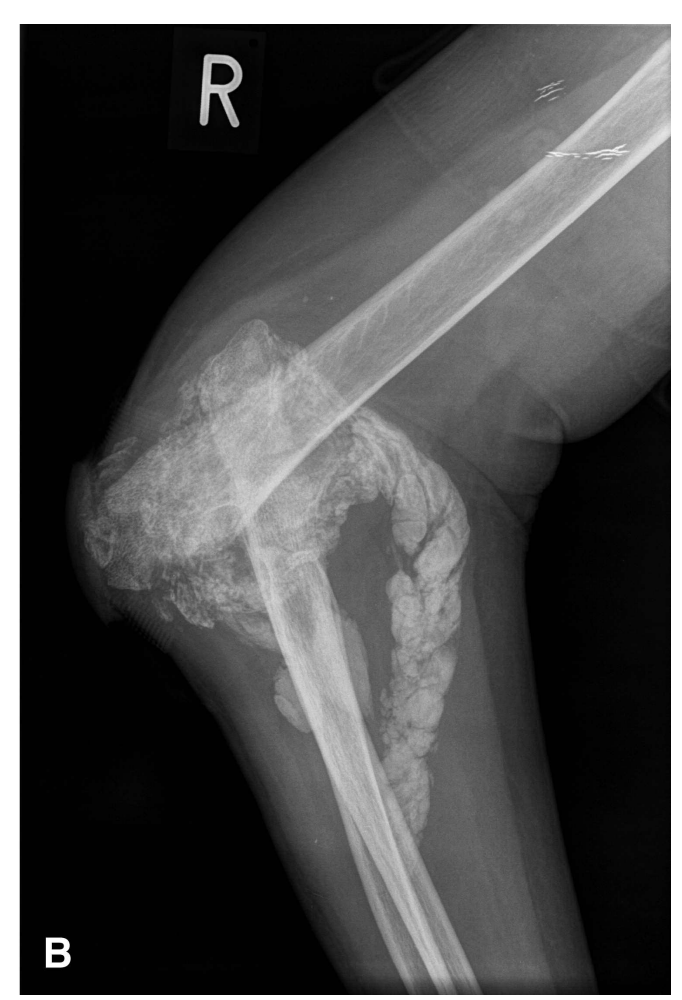

mation. There was no clinical evidence of interstitial lung disease.

Anti-Jo1 antibody is typically associated with the antisynthetase syndrome, which is characterized by inflammatory myositis, arthritis, Raynaud phenomenon, mechanic's hands, and interstitial lung disease. Calcinosis and deforming arthropathy, particularly of the hands, associated with anti-Jo1 antibody have been reported infrequently in the literature ${ }^{1,2}$. However, to our knowledge, such striking calcinosis and rapidly progressive destruction of the elbow joint has not been reported before.

\section{REFERENCES}

1. Delbrel X, Schaeverbeke T, Lifermann F, Dehais J. Arthropathy associated with anti-Jo-1 antibody. Joint Bone Spine 2001; $68: 166-9$

Personal non-commercial use only. The Journal of Rheumatology Copyright $\odot$ 2015. All rights reserved. 

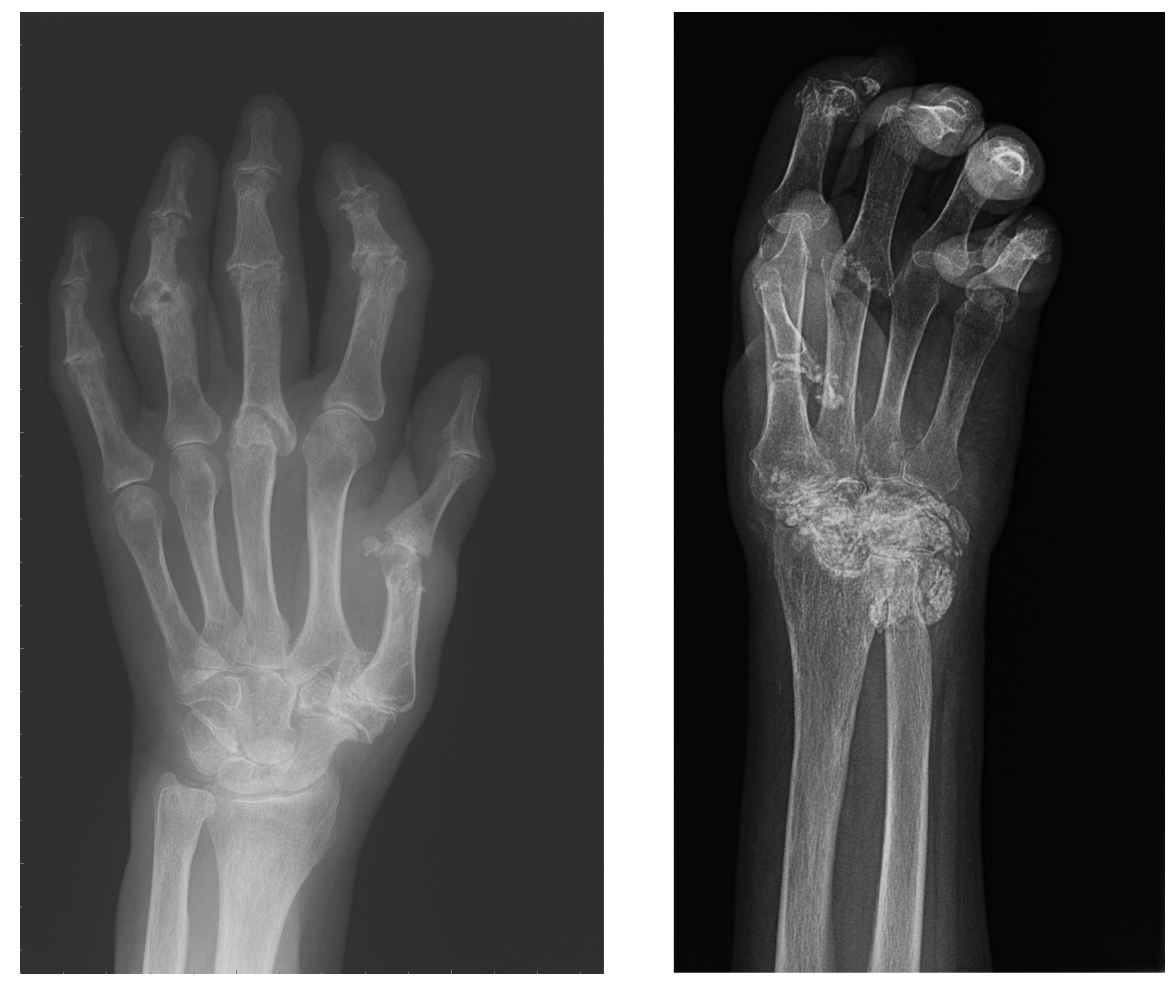

Figure 2. Radiographs of the left hand in 2008 (left panel) and 2012 show markedly progressive calcinosis and destructive arthropathy.

2. Queiro-Silva R, Bañegil I, de Dios-Jiménez de Aberásturi JR, Belzunegui-Otano J, González-Beneitez C, Figueroa-Pedrosa M. Periarticular calcinosis associated with anti-Jo-1 antibodies sine myositis. Expanding the clinical spectrum of the antisynthetase syndrome. J Rheumatol 2001;28:1401-4. 